\title{
High Temperature Asymptotics in Terms of Heat Kernel Coefficients: Boundary Conditions with Spherical and Cylindrical Symmetries
}

\author{
M. Bordag* \\ Universität Leipzig, Institut für Theoretische Physik \\ Augustusplatz 10, 04109 Leipzig, Germany \\ V.V. Nesterenko ${ }^{\dagger}$, I.G. Pirozhenko ${ }^{\ddagger}$ \\ Bogoliubov Laboratory of Theoretical Physics \\ Joint Institute for Nuclear Research, Dubna, 141980, Russia
}

(October 27, 2018)

\begin{abstract}
The high temperature asymptotics of the Helmholtz free energy of electromagnetic field subjected to boundary conditions with spherical and cylindrical symmetries are constructed by making use of a general expansion in terms of heat kernel coefficients and the related determinant. For this, some new heat kernel coefficients and determinants had to be calculated for the boundary conditions under consideration. The obtained results reproduce all the asymptotics derived by other methods in the problems at hand and involve a few new terms in the high temperature expansions. An obvious merit of this approach is its universality and applicability to any boundary value problem correctly formulated.
\end{abstract}

The Casimir calculations at finite temperature prove to be a nontrivial problem specifically for boundary conditions with nonzero curvature. Investigation of the high temperature limit, i.e. the classical limit, in this problem is of independent interest [1]. For this goal a powerful method of the zeta function technique and the heat kernel expansion can be used. It is essential that for obtaining the high temperature asymptotics of the thermodynamic characteristics it is sufficient to know the heat kernel coefficients and the determinant for the spatial part of the operator governing the field dynamics. This determinant is the derivative of its zeta function in zero, whereas the Casimir energy at zero temperature is given by this zeta function in the point $-\frac{1}{2}$. This is an essential merit of this approach. This high temperature expansion in terms of the heat kernel coefficients and the zeta function determinant is known for a long time [2], but did not receive the due attention and has to our knowledge not been used in the case of boundary conditions. The present study seeks to fill the gap in this area and to demonstrate the universality and efficiency of this approach by treating a number of nontrivial boundary conditions for the electromagnetic field. We present here only the basic results of our calculations.

Starting point in our consideration is the general high temperature expansion of the free energy initially given in [2] and recently discussed in [3], Sec. 5.1.3,

$$
\begin{aligned}
F(T) \simeq & -\frac{T}{2} \zeta^{\prime}(0)+a_{0} \frac{T^{4}}{\hbar^{3}} \frac{\pi^{2}}{90}-\frac{a_{1 / 2} T^{3}}{4 \pi^{3 / 2} \hbar^{2}} \zeta_{R}(3)-\frac{a_{1}}{24} \frac{T^{2}}{\hbar}+\frac{a_{3 / 2}}{(4 \pi)^{3 / 2}} T \ln \frac{\hbar}{T}-\frac{a_{2}}{16 \pi^{2}} \hbar\left[\ln \left(\frac{\hbar}{4 \pi T}\right)+\gamma\right] \\
& -\frac{a_{5 / 2}}{(4 \pi)^{3 / 2}} \frac{\hbar^{2}}{24 T}-T \sum_{n \geq 3} \frac{a_{n}}{(4 \pi)^{3 / 2}}\left(\frac{\hbar}{2 \pi T}\right)^{2 n-3} \Gamma(n-3 / 2) \zeta_{R}(2 n-3) .
\end{aligned}
$$

Here $\gamma$ is the Euler constant and $\zeta_{R}(s)$ is the Riemann zeta function. The quantities under the logarithm sign in expansion (11) are dimensional, but upon collecting similar terms with account for the logarithmic ones in $\zeta^{\prime}(0)$ it is easy to see that finally the logarithm function has a dimensionless argument.

It is worth noting that the zeta function determinant, i.e., $\zeta^{\prime}(0)$, does not enter the asymptotic expansion for the internal energy which is completely defined only by the heat kernel coefficients. In view of this, the first term in the asymptotics of the free energy in Eq. (11) is referred to as a pure entropic contribution [1]. Its physical origin is till now not elucidated. The entropic term is a pure classical quantity because it does not involve the Planck constant $\hbar$. This classical contribution to the asymptotics seems to be derivable without appealing to the notion of quantized electromagnetic field.

\footnotetext{
*E-mail: Michael.Bordag@itp.uni-leipzig.de

${ }^{\dagger}$ E-mail: nestr@thsun1.jinr.ru

${ }^{\ddagger}$ E-mail: pirozhen@thsun1.jinr.ru
} 
The heat kernel coefficients needed for construction of the expansion (11) will been calculated as the residua of the corresponding zeta functions. For the boundary conditions under consideration the explicit expressions for the zeta functions have been derived in our papers [4,5]. The zeta function determinants involved in Eq. (1) are calculated in Ref. [6] using the methods of Ref. [7]. For a simple calculation of the relevant heat kernel coefficients see also Ref. [8]

A perfectly conducting spherical shell of radius $R$ in vacuum. The first six heat kernel coefficients in this problem are:

$$
a_{0}=0, \quad a_{1 / 2}=0, \quad a_{1}=0, \quad \frac{a_{3 / 2}}{(4 \pi)^{3 / 2}}=\frac{1}{4}, \quad a_{2}=0, \quad \frac{a_{5 / 2}}{(4 \pi)^{3 / 2}}=\frac{c^{2}}{160 R^{2}}
$$

Furthermore

$$
a_{j}=0, \quad j=3,4,5, \ldots
$$

The exact value of $\zeta^{\prime}(0)$ is derived in [6]

$$
\zeta^{\prime}(0)=\left(\frac{1}{2}-\frac{\gamma}{2}+\frac{7}{6} \ln 2+6 \zeta_{R}^{\prime}(-1)\right)+\left(-\frac{5}{8}+\frac{1}{2} \ln \frac{R}{c}+\ln 2+\frac{\gamma}{2}\right)=0.38429+\frac{1}{2} \ln \frac{R}{c}
$$

As a result we have the following high temperature asymptotics of the free energy

$$
F(T)=-\frac{T}{4}\left(0.76858+\ln \tau+\frac{1}{960 \tau^{2}}\right)+\mathcal{O}\left(T^{-3}\right)
$$

where $\tau=R T /(\hbar c)$ is the dimensionless 'temperature'. The expression (5) exactly reproduces the asymptotics obtained in Ref. [9] by making use of the multiple scattering technique (see Eq. (8.39) in that paper).

A compact ball with $c_{1}=c_{2}$. In this case the spherical surface delimits the media with "relativistic invariant" characteristics i.e., the velocity of light is the same inside and outside the ball $[10$. Here there naturally arises a dimensionless parameter [1]

$$
\xi^{2}=\left(\frac{\varepsilon_{1}-\varepsilon_{2}}{\varepsilon_{1}+\varepsilon_{2}}\right)^{2}=\left(\frac{\mu_{1}-\mu_{2}}{\mu_{1}+\mu_{2}}\right)^{2},
$$

where $\varepsilon_{1}$ and $\varepsilon_{2}\left(\mu_{1}\right.$ and $\left.\mu_{2}\right)$ are permittivities (permeabilities) inside and outside the ball. As usual we perform the calculation in the first order of the expansion with respect to $\xi^{2}$.

The zeta function for this boundaries, obtained in Ref. [ 4 , affords the exact values of heat kernel coefficients up to $a_{3}$

$$
a_{0}=a_{1 / 2}=a_{1}=0, \quad a_{3 / 2}=2 \pi^{3 / 2} \xi^{2}, \quad a_{2}=0, \quad a_{5 / 2}=0, \quad a_{3}=0 .
$$

The zeta determinant in this problem turns out to be given by multiplication of the content of the second parentheses in Eq. (4) by $\xi^{2}$

$$
\zeta^{\prime}(0)=\xi^{2}\left(0.35676+\frac{1}{2} \ln \frac{R}{c}\right) .
$$

The high temperature asymptotics for free energy reads

$$
F(T)=-\xi^{2} \frac{T}{4}\left(\gamma+\ln 4-\frac{5}{4}+\ln \tau\right)+\mathcal{O}\left(T^{-3}\right)=-\xi^{2} \frac{T}{4}(0.71352+\ln \tau)+\mathcal{O}\left(T^{-3}\right) .
$$

The asymptotics (9) completely coincide with analogous formula obtained in Refs. 12,13 by the mode summation method combined with the addition theorem for the Bessel functions.

A pure dielectric ball. In this case the heat kernel coefficients $a_{0}$ and $a_{1 / 2}$ prove to be nonvanishing [14]. Their contributions to the high temperature asymptotics should be removed because the Stefan-Boltzmann constant should not be renormalized. For the next coefficients we have

$$
a_{1}=a_{2}=0, \quad a_{3 / 2}=\pi^{3 / 2} \Delta n^{2}, \quad a_{5 / 2}=0,
$$

where $\Delta n=n_{1}-n_{2}=n_{1} n_{2}\left(c_{2}-c_{1}\right) / c \simeq\left(c_{2}-c_{1}\right) / c, n_{i}$ and $c_{i}$ are the refractive index and the velocity of light inside $(i=1)$ and outside $(i=2)$ the ball, and $c$ is the velocity of light in the vacuum. It is assumed that $c_{1}$ and $c_{2}$ 
differ from $c$ slightly, therefore $c_{2}-c_{1}$ and $\Delta n$ are small quantities. Making use of the technique developed in Ref. [7] one obtains the following expression for the zeta function determinant [6]

$$
\zeta^{\prime}(0)=\frac{\Delta n^{2}}{4}\left(-\frac{7}{8}+\ln \frac{R}{c}+\ln 4+\gamma\right)
$$

The high temperature asymptotics of free energy reads

$$
F(T)=-\frac{\Delta n^{2}}{8} T\left(\ln 4 \tau+\gamma-\frac{7}{8}\right)+\mathcal{O}\left(T^{-2}\right) .
$$

It is worth comparing these results with analogous asymptotics obtained by different methods. In Ref. [12] at the beginning of calculations the first term of expansion of the internal energy was derived. The subsequent integration of the thermodynamic relation gave the correct coefficient of the logarithmic term in the asymptotics of free energy (12). Barton [15] managed to deduce the asymptotics (12) except for the contributions proportional to negative powers of $T$. One should keep in mind that our parameter $\Delta n$ corresponds to $2 \pi \alpha n$ in the notations of Ref. 115. The asymptotics (12) contain the $R$-independent terms. As far as we know the physical meaning of such terms remains unclear.

A perfectly conducting cylindrical shell. The heat kernel coefficients are

$$
a_{0}=a_{1 / 2}=a_{1}=a_{2}=0, \quad \frac{a_{3 / 2}}{(4 \pi)^{3 / 2}}=\frac{3}{64 R}, \quad \frac{a_{5 / 2}}{(4 \pi)^{3 / 2}}=\frac{153}{8192} \frac{c^{2}}{R^{3}} .
$$

The zeta function determinant in this problem is calculated in [6]

$$
\zeta^{\prime}(0)=\frac{0.45711}{R}+\frac{3}{32 R} \ln \frac{R}{2 c} .
$$

The free energy behavior at high temperature is the following

$$
F(T)=-\frac{T}{R}\left(0.22856+\frac{3}{64} \ln \frac{\tau}{2}-\frac{51}{65536 \tau^{2}}\right)+\mathcal{O}\left(T^{-3}\right) .
$$

The high temperature asymptotics of the electromagnetic free energy in presence of perfectly conducting cylindrical shell was investigated in Ref. [9]. To make the comparison handy let us rewrite their result as follows

$$
F(T) \simeq-\frac{T}{R}\left(0.10362+\frac{3}{64 R} \ln \frac{\tau}{2}\right) .
$$

The discrepancy between the terms linear in $T$ in Eqs. $(15)$ and $(16)$ is due to the double scattering approximation used in Ref. [9] (see also below). Our approach provides an opportunity to calculate the exact value of this term (see Eq. (15)).

A compact infinite cylinder with $c_{1}=c_{2}$. By making use of the relevant zeta function found in Ref. [4] one obtains the heat kernel coefficients

$$
\begin{gathered}
a_{0}=a_{1 / 2}=a_{1}=a_{2}=a_{j}=0, j=3,4, \ldots, \\
\quad \frac{a_{3 / 2}}{(4 \pi)^{3 / 2}}=\frac{3 \xi^{2}}{64 R}, \frac{a_{5 / 2}}{(4 \pi)^{3 / 2}}=\xi^{2} \frac{c^{2}}{R^{3}} \frac{45}{8192} .
\end{gathered}
$$

The corresponding zeta function determinant is calculated in Ref. [6]

$$
\zeta^{\prime}(0)=\frac{\xi^{2}}{R}\left(0.20483+\frac{3}{32} \ln \frac{R}{2 c}\right) .
$$

Now we can write the high temperature asymptotics for free energy

$$
F(T)=-\xi^{2} \frac{T}{R}\left(0.10242-\xi^{2} \frac{3}{64} \ln \tau+\frac{15}{65536 \tau^{2}}\right)+\mathcal{O}\left(T^{-3}\right) .
$$

Putting in Eq. (19) $\xi^{2}=1$ we arrive at the double scattering approximation for a perfectly conducting cylindrical shell (16). 
A pure dielectric cylinder. In this problem the first three heat kernel coefficients prove to be nonzero [5]. Their contributions to the high temperature asymptotics should be canceled by respective redefinition of the parameters in the general expression for free energy of such a cylinder (in the same way as it has been done for a pure dielectric ball). The next coefficients are given by

$$
a_{2}=0, \quad a_{3 / 2}=\frac{3 \pi^{3 / 2}}{16 R c_{2}^{2}}\left(c_{1}-c_{2}\right)^{2}, \quad \frac{a_{5 / 2}}{(4 \pi)^{3 / 2}}=\frac{857}{61440} \frac{\left(c_{1}-c_{2}\right)^{2}}{R^{3}} .
$$

On this basis we can write the high temperature asymptotics for internal energy in the problem at hand

$$
U(T)=\Delta n^{2} \frac{3}{128} \frac{T}{R}\left(1-\frac{857}{17280 \tau^{2}}\right)+\mathcal{O}\left(T^{-2}\right),
$$

where $\Delta n=n_{1}-n_{2} \simeq\left(c_{2}-c_{1}\right) / c$. In view of considerable technical difficulties we shall not calculate the zeta function determinant for a pure dielectric cylinder. We recover the respective asymptotics for free energy by integrating the appropriate thermodynamic relation using Eq. 21). Pursuing this way we introduce a new constant of integration $\alpha$ that remaines undetermined

$$
F(T)=-\Delta n^{2} \frac{3}{128} \frac{T}{R}\left(\alpha+\ln \tau+\frac{857}{34560 \tau^{2}}\right)+\mathcal{O}\left(T^{-2}\right) .
$$

To sum up we have demonstrated efficiency and universality of the high temperature expansions in terms of the heat kernel coefficients for the Casimir problems with spherical and cylindrical symmetries. All the known results in this field are reproduced in a uniform approach and in addition a few new asymptotics are derived (an exact asymptotics for a perfectly conducting cylindrical shell, for a compact cylinder with $c_{1}=c_{2}$, and for a pure dielectric infinite cylinder).

As the next step in the development of this approach one can try to retain the terms exponentially decreasing when $T \rightarrow \infty$. These corrections are well known, for example, for thermodynamic functions of electromagnetic field in the presence of perfectly conducting parallel plates [16]. In order to reveal such terms, first of all the exponentially decreasing corrections should be retained in the standard asymptotic expansion for the heat kernel.

As in all the Casimir calculations taking into account the material characteristics of the boundaries, in the approach under consideration the renormalization procedure should be formulated exactly in the framework of the high temperature expansions (1). The explicit divergencies are not encountered here, but nevertheless the renormalization should be carried out as it was shown in this paper when considering a pure dielectric ball and dielectric cylinder.

[1] J. Feinberg, A. Mann, and M. Revzen, Ann. Phys. (N.Y.) 288 (2001) 103.

[2] J. S. Dowker and G. Kennedy, J. Phys. A 11 (1978) 895.

[3] M. Bordag, U. Mohideen, and V. M. Mostepanenko, Phys. Rep. 353 (2001) 1; quant-ph/0106045.

[4] G. Lambiase, V. V. Nesterenko, and M. Bordag, J. Math. Phys. 40 (1999) 6254.

[5] M. Bordag and I. G. Pirozhenko, Phys. Rev. D 64 (2001) 025019.

[6] M. Bordag, V. V. Nesterenko, and I. G. Pirozhenko, hep-th/0107024, to be published in Phys. Rev. D.

[7] M. Bordag, B. Geyer, K. Kirsten, and E. Elizalde, Commun. Math. Phys. 179 (1996) 215.

[8] M. Bordag, K. Kirsten, E. Elizalde, J. Math. Phys. 37 (1996) 895.

[9] R. Balian and B. D. Duplantier, Ann. Phys. (N.Y.) 112 (1978) 165.

[10] I. Brevik, Phys. Rep. 52, 133 (1979).

[11] I. Brevik, V. V. Nesterenko, and I. G. Pirozhenko, J. Phys. A 31 (1998) 8661.

[12] V. V. Nesterenko, G. Lambiase, and G. Scarpetta, Phys. Rev. D 64 (2001) 025013/8.

[13] I. Klich, J. Feinberg, A. Mann, and M. Revzen, Phys. Rev. D 62 (2000) 045017.

[14] M. Bordag, K. Kirsten and D. Vassilevich, Phys. Rev. D 59 (1999) 085011.

[15] G. Barton, Phys. Rev. A 64 (2001) 032103.

[16] G. Plunien, B. Muller, W. Greiner, Phys. Rep. 134, (1986) 87. 